\title{
Supporting medical decision making with argumentation tools 敞, 论的
}

\author{
Jingyan $\mathrm{Lu}^{\mathrm{a}, *}$, Susanne P. Lajoie ${ }^{\mathrm{b}}$ \\ a Division of Information and Technology Studies, Faculty of Education, The University of Hong Kong, Pokfulam Road, Hong Kong \\ ${ }^{\mathrm{b}}$ Department of Educational and Counselling Psychology, McGill University, 3700 McTavish Street, Montreal, Que., Canada H3A 1 Y2
}

\section{A R T I C L E I N F O}

Article history:

Available online 27 June 2008

\section{Keywords:}

Collaborative learning

Argumentation

Problem solving

Medical decision-making

Computer-supported collaborative learning

Argumentation tools

Cognitive tools

Discourse analysis

\begin{abstract}
A B S T R A C T
This study investigated the collaborative decision-making and communicative discourse of groups of learners engaged in a simulated medical emergency in two conditions. In one condition subgroups used a traditional whiteboard (TW group) to document medical arguments on how to solve a medical emergency. In the other condition subgroups used interactive whiteboards (IW group) where they could document their medical arguments by using a structured template for constructing, annotating and sharing arguments. The discourse of each group was coded with respect to decision-making and communicative activities. The research goal was to identify relationships between the decision-making and collaborative discourse under the two conditions. The IW condition differed from the TW condition in that participants engaged in more adaptive decision-making behavior early on in the intervention. This early engagement led to shared understandings and subsequently to more effective patient management. Group differences were also found in the types of collaborative discourse and the IW groups produced more productive argumentation.
\end{abstract}

(c) 2008 Elsevier Inc. All rights reserved.

\footnotetext{
Paper submitted to Contemporary Educational Psychology.

This research was supported in part by a Ph.D. fellowship from the Fonds Québécois de la recherche sur la société et la culture (FQRSC) to the first author while she completed her Ph.D. with the second author at McGill who also funded this work with her grant from the Canadian Social Sciences and Humanities Research Council. We would like to thank Dr. Jeffrey Wiseman for providing his innovative teaching activity with us, along with his classrooms, and his great insights into medical instruction. We also thank Mr. Tom Patrick for his tremendous contributions with the data collection process as well as his openness to discussing ideas in the preparation of this manuscript.

* Corresponding author. Fax: +852 25471924.

E-mail addresses: jingyan@hkucc.hku.hk (J. Lu), susanne.lajoie@mcgill.ca (S.P. Lajoie).
} 


\section{Introduction}

Medical emergencies frequently require medical teams to work together collaboratively to save patient lives. Expertise is distributed among the medical team and collaboration requires communicating effectively so that knowledge about the patient can be shared with others so that appropriate decisions and actions are taken to help the patient. Medical dialogue in such situations consists of argumentation, where physicians formulate arguments that consist of a list of patient problems based on the evidence that they have collected. The argument includes presentations or reviews of patient history, symptoms, diagnostic test results and other evidence needed to make a decision about diagnosis and patient management. These arguments are used to persuade the rest of the medical team to act in a particular way. Medical arguments are typically co-constructed as information is shared and updated based on the patient's reactions to care. This article explores how medical students formulate medical arguments in the context of collaborative decision making in emergency medicine. We explore these situations where technology is designed to support argumentation and in situations without technology support.

Our goal is to support medical groups, who work collaboratively in their decision making about patient care. Medical arguments are analyzed by describing the types of communication and decision-making activities taken in the creation of a joint problem space in a simulated naturalistic decision making context where students learn to manage patient cases. Collaboration is examined in groups that are supported with technology and in groups that are not supported with technology. The technology group is provided with laptops that are used with an interactive whiteboard (IW). An argumentation tool was designed that includes menus that support the documentation of a medical argument. It is hypothesized that structuring the medical argument with this tool supports argumentation by providing external frames for constructing knowledge (Roschelle \& Teasley, 1995) and by visually representing arguments which ultimately lead to more coherent, focused and productive discussions (Hron, Hesse, Cress, \& Giovis, 2000). This study examines whether or not such argumentation tools can make a difference in decision-making and communicative activities in a simulated medical emergency situation.

In the section below we provide a short review of research that led to this study. We begin with a review of collaborative learning and the importance of argumentation to the learning process. The collaborative nature of emergency medicine can be studied in naturalistic decision making contexts where the team approach to decision making about patient care is examined to determine the nature of shared goals, background knowledge, effective communication and situational awareness required to make accurate decisions in a dynamically emerging setting. We include a discussion of naturalistic decision making research since we are examining argumentation as a step toward team decision making about patient care. Finally, we discuss the literature on technology rich learning environments with respect to the tools designed to support argumentation.

\subsection{Collaborative learning and argumentation}

Collaborative learning has long been recognized as an effective pedagogical method that can promote deeper level learning, critical thinking, shared understanding, and long term retention of the learned material (e.g., Johnson \& Johnson, 1999; Slavin, 1995). Collaborative learning has been variously conceptualized in such learning theories as social constructivism (Dosie \& Mugny, 1984; Piaget, 1970), situated learning (Brown, Collins, \& Duguid, 1989; Lave, 1991), shared cognition (Resnick, Levine, \& Teasley, 1991), and distributed cognition (Salomon, 1993). Each of these theories adopts a unique perspective on collaborative learning. For example, the social constructivist tends to focus on how social conflicts stimulate learning within a group context; situated cognition emphasizes learning as a process of participation as opposed to acquisition; shared cognition emphasizes the building and maintaining of shared understanding in authentic contexts; and distributed cognition emphasizes the distribution of expertise among learners, environments, and artifacts. Despite having different foci, these theories all conceive of learning as a collaborative activity, during which argumentation plays important roles (Salomon, 1993) from exposing social conflicts (Veerman, 2003), promoting commu- 
nity discourse (Schwarz \& Glassner, 2003), to facilitating shared understanding across group members (Teasley \& Roschelle, 1993).

Argumentation has been viewed as one of the major mechanisms of collaborative learning as it plays an important role in "confronting cognitions" (Andriessen, Baker, \& Suthers, 2003) during collaborative problem solving and knowledge building. Cognitions are usually viewed as the internal representation of the mind and can only be demonstrated in certain indirect ways, such as verbal statements, body language, or diagrams and pictures. Students who engage in the argumentation process show more productive collaboration because they are usually presented with conflicts and need to negotiate for agreements in order to arrive at shared solutions and ideas (Chan, Burtis, \& Beretier, 1997; Chi, Slotta, \& de Leeuw, 1994). During collaborative interactions cognitive conflicts arise and inadequate reasoning is exposed and consequently higher-quality understanding can emerge. Meanwhile, explanations are provided to each other regarding the conflicts so that knowledge and opinions can be constructed and co-constructed (Slavin, 1995). Technology rich learning environments that provide cognitive tools to support learning do exist (Jonassen, 1995; Jonassen \& Carr, 2000; Jonassen \& Reeves, 1996; Lajoie, 1993; Lajoie, 2000; Lajoie \& Azevedo, 2006; Salomon, Perkins, \& Globerson, 1991 ) and guide this research in the design of tools to support the argumentation process. Traditional studies of argumentation have looked at claims, evidence, and rebuttals (Kuhn, 1991) whereas recent studies of argumentation are less structured in their definitions (Kanselaar et al., 2003). Argumentation can be a self-explanation (Chi \& VanLehn, 1991) or reasoning process taking place within one individual or it can happen between two or more people in the form of negotiating the meaning of concepts (Baker, 1994), problem-solving solutions (Baker, 2003), or justifying or explaining answers to questions (Jermann \& Dillenbourg, 2003; Nussbaum \& Sinatra, 2003; Webb, Troper, \& Fall, 1995). Definitions of argumentation range from traditionally defined activities such as giving reasons, persuading, convincing, or demonstrating a point of view (Carr, 2003) to recently promoted activities such as conceptual change, co-elaboration and co-construction of knowledge (Baker, 1994; Baker, 1995; Veerman, 2003).

Argumentative interactions in a collaborative situation of this study take place in the context of making decisions about patient diagnosis or management. For example, an argumentative interaction may occur when deciding which evidence should be prioritized and which solution is the best among other choices. In collaborative medical decision-making, argumentative interactions may focus on which symptoms are most urgent and should get immediate attention and management. Thus, argumentation in this collaborative problem solving environment can be seen more as a co-elaboration of ideas or negotiation of meaning, and persuasion for a particular point of view (Baker, 2003). Accepting or rejecting a point of view requires certain types of knowledge about the problem which is the basis for negotiation of meaning and elaboration of knowledge. These negotiation and co-elaboration processes change the epistemological status of participants so that they are better able to accept or reject certain points of view (Baker, 2003). The next section examines argumentation and collaborative decision-making in naturalistic as opposed to contrived settings. Naturalistic decision-making (NDM) is different than classical decision-making where individuals are studied in settings where decisions are made by comparing and choosing among a constrained set of options (Beach \& Lipshitz, 1993).

\subsection{Naturalistic decision-making}

The naturalistic decision-making (NDM) literature is vast in that it covers a variety of real-world situations where decisions are often made based on incomplete or ambiguous information (Zsambok, 1997). Whereas early studies of NDM were restricted to how military personnel make decisions under extreme time pressure (Klein, Calderwood, \& Clinton-Cirocco, 1986), more recent research include naturalistic decision making in commercial and military aviation (Orasanu \& Salas, 1993), anesthesiology (Gaba, 1992), fire fighting (Cannon-Bowers \& Salas, 1998) and emergency medical care situations (Lajoie, Azevedo, \& Fleiszer, 1998; Leprohon \& Patel, 1995) where personnel make high stakes decisions in dynamic high risk settings.

The dynamic, complex and high risk nature of critical care imposes intense cognitive demands on doctors who have little time to make decisions and must stabilize patients prior to further diagnosis. Consequently, in order to characterize the features of real-world emergency procedures, NDM 
researchers must focus on more action-oriented research. Lajoie and colleagues (1998) investigated the clinical decision-making actions of surgical nurses and revealed the variability in nurses' decision-making paths. These findings can then be used to inform instructional practice and training by designing learning environments (with or without technology) that support real world decision making (Lajoie, 2000; Lajoie \& Azevedo, 2000; Lajoie et al., 1998).

NDM often involves teams who share mental models as they work together to make decisions. "Shared mental models" refers to a shared understanding of task goals and task-relevant knowledge that guide team members to work effectively by helping them define problems, acceptable outcomes, and roles of team members (Cannon-Bowers, Salas, \& Converse, 1993). Explicit communication is needed to develop such models and to achieve a shared understanding of the problems at hand. Because of the changing nature of NDM, members must also communicate their situational awareness given the dynamic nature of their task (Cannon-Bowers et al., 1993). Effective communication during NDM facilitates the development of shared understandings. In emergent medical situations, doctors must assess and communicate rapidly changing patient conditions to other medical personnel for various reasons. This in turn facilitates the construction of shared understandings of goals, plans, and actions for managing patients. Communication is an important aspect of collaborative decision-making. Without communication it would be impossible to establish a shared mental model or shared understanding of the task at hand. This study examines communication in the context of decision making as it leads to a shared understanding of the patient and how such understanding guides actions in emergent medical situations.

The emergence of computer technology broadens the channels for communication from traditional face-to-face situations to distributed online environments, and presents new features and possibilities that can support argumentation that cannot be realized in face-to-face verbal situations. These new possibilities are presented in the next section, where we describe how computers can be designed with argumentation tools to support learning.

\subsection{Argumentation tools}

Technology-rich learning environments need to be designed based on learning theories intended to support, transform or extend learning for specific situations (Lajoie, 2007; Lajoie \& Azevedo, 2006). Computers can be designed with cognitive tools to support learning and for the purpose of this article we focus on supporting collaborative decision making by fostering the development of a medical argument. Computer environments support argumentation by helping learners elaborate, explain, reflect on and track their ideas (Lajoie \& Azevedo, 2006). Computer-based argumentation tools mediate communication, structure interaction, guide argumentation and represent arguments (Andriessen et al., 2003). These features are described below.

Technology driven argumentation tools can be designed to mediate both synchronous and asynchronous communication. The immediacy of synchronous interactions can motivate participants to engage in and carry on interpersonal negotiations (Wang \& Chen, 2006). Learners can test and refine what they are learning in a community that offers immediate feedback (Jonassen, 2000; Jonassen \& Carr, 2000). Asynchronous communication can occur using tools like WebCT where students engage in dialogue at their convenience by adding to on-line discussion forums which can promote both communities of inquiry and cognitive apprenticeship (Lajoie et al., 2006). Asynchronous communication allows students to co-construct and share knowledge across time and space (Edelson \& O'Neill, 1994; Scardamalia \& Bereiter, 1996; Winne, 2006) or allows students to share perspectives, establish relationships, seek assistance (Chong, 1998), distinguish alternative views on scientific topics (Hoadley \& Linn, 2000), and promote sustained and in-depth discussions (Guzdial \& Turns, 2000).

Argumentation tools structure interactions by orienting participants with respect to subject matter by supporting greater coherence in discussions, increasing focus on topics and consequently reduce off-task talking (Hron et al., 2000). Structuring can be achieved through communication acts (Baker, 2003), sentence openers (Baker \& Lund, 1997; Hirsch, Saeedi, Cornillon, \& Litosseliti, 2004), posting notes and making comments (Fischer, Bruhn, Grasel, \& Mandl, 2002; Scardamalia \& Bereiter, 1996), or representing multiple opinions that scaffold students to express their own opinions and integrate the opinions of others (Hoadley \& Linn, 2000). 
Three management tools guide argumentation by supporting the functions of mirroring, metacognition, and advising (Jermann, Soller, \& Muehlenbrock, 2001; Reimann, 2003). Mirroring tools help manage collaboration by tracking interactions and collaborative performance among group members. Interaction and performance data can be collected and analyzed for further comparison and guidance. Metacognitive tools require learners to first construct models of interactions and then to compare them to desired states. Advising tools are used to intervene, advise and guide learners after collaboration data have been analyzed.

Visual representations of arguments can serve as an external frame for constructing collaborative knowledge building and problem solving (Roschelle \& Teasley, 1995). Such visualizations could be in the form of concept mapping (Stoyanova \& Kommers, 2002), diagramming (van Boxtel \& Veerman, 2001), and text (Hoadley \& Linn, 2000). Visualizations of an argument can foster abstract conceptual understanding by showing the relationship between data and evidence (Suthers \& Hundhausen, 2001) and consequently induce higher level discourse and possibly foster conflict-oriented consensus building (Fischer et al., 2002).

Given the importance of argumentation skills to support collaborative learning, we describe argumentation tools designed to support medical students decision-making in the context of simulated medical emergencies. These tools are described in the context of the research study. This study differs from many studies on medical cognition in that it focuses on: (a) NDM in a simulated emergency setting as opposed to a formalized laboratory-based setting, (b) collaborative decision making as opposed to individual learning, and (c) monitoring and managing patients rather than simply diagnosing patients. This study aims to investigate the following questions pertaining to students making decisions without technology support and those provided with argumentation tools via technology: (a) do students under the two conditions differ in their decision making activities? (b) do students under the two conditions differ in their communicative activities? (c) what are the characteristics of online annotation, and (d) if student decision-making and communicative activities differ under the two conditions, why do they differ? The methods for each research question are described below.

\section{Methods}

\subsection{Subjects and design}

Third year medical students were recruited from an internal medicine rotation. Two groups of seven students volunteered to participate in this study. All students received the same clinical training at the same large urban teaching hospital and their background knowledge before participating in this study was assumed to be comparable given the curriculum that was followed and the strict entry requirements of the medical school. Students were debriefed on the purpose of the research at the completion of the study.

The students attended two clinical teaching sessions one at the beginning and one at end of their two-month rotation. The teacher organized students into three subgroups based on seating proximity. Group members in each subgroup were kept consistent during the two teaching sessions. Both groups were asked to solve a learning activity called the 'deteriorating patient' designed by Dr. Jeffrey Wiseman in both teaching sessions (personal communication, November 24, 2005). One group of students used a traditional whiteboard (hereafter called TW group) whereas the other using an interactive whiteboard (hereafter called IW group) while solving the patient problem. The deteriorating patient activity is described below since it serves as learning context where decision-making and communication are examined.

\subsection{Deteriorating patient activity}

The deteriorating patient activity simulates actual medical emergencies that medical students will encounter in Emergency Rooms in most hospitals. Participants are placed in situations where they must make decisions quickly based on the patient's rapidly deteriorating medical condition. Each teaching session is an hour long and consists of three stages. First, the teacher explains the activity, 
the rules and roles that students will play. In the second stage, students solve the problem, and in the third stage, the teacher summarizes the case and debriefs the students in how they should have managed the emergency.

For the purpose of this study, we concentrate on stage two where students are engaged in problem solving. The teacher presents the patient case and asks for a volunteer to start solving the problem. When this student runs into difficulty, the teacher tells him or her to call the junior resident (played by another student). When the junior resident reaches an impasse $s /$ he calls the senior resident who is played by a third student. In this way the activity simulates the operation of a real medical emergency. The teacher plays two roles, the deteriorating patient and the duty nurse. As the deteriorating patient, the teacher acts out (rather dramatically) the patient's fluctuating physical conditions. As the duty nurse he constantly updates patient's fluctuating vital signs and symptoms. Students ask the nurse questions about the patient and based on his answers determine what to do next. The nurse carries out their orders, reports results and delivers updates on the current state of the patient's vital signs and symptoms.

When we first observed the teacher conducting this activity we saw students take turns to solve the problem individually. Unfortunately only half of the students were able to participate in the role-play experience due to time limitations. The other half of the students only observed. We altered this activity to provide opportunities for greater participation by creating two conditions where students worked collaboratively to solve cases. Students were divided into sub-groups and each sub-group took turns interacting with the teacher so that all the students had a chance at solving the problem. The TW group used a traditional (front-of-the-class) whiteboard to document the medical argument about how to solve the medical emergency. The IW group was supported by technology, more specifically they had laptops that interfaced with the IW and each sub-group could add information to the medical argument by using a structured template for building, annotating and sharing arguments.

\subsection{Procedures}

Both the TW and IW groups were presented with a patient case and had several minutes to discuss it and form questions to ask the teacher in order to collect additional patient information and to formulate plans and actions for stabilizing the patient. The sub-groups took turns interacting with the teacher to collect information about the patient. IW subgroups used laptops that interfaced with the IW and their screens displayed the information that the teacher wrote on the patient chart displayed on the traditional whiteboard. Since all IW subgroups were networked to the IW they were able to annotate the patient chart on their laptops and see each other's annotations. Both the IW and TW groups followed the same rules interacting with the teacher. When one sub-group was solving the problem, the other groups could not interfere. Consequently, their opportunities for contributing to the verbal interaction are equivalent.

\subsection{Argumentation tools to support collaborative decision-making}

The IW electronically captures notes and images that are written on the traditional whiteboard in real time using an eBeam ${ }^{\mathrm{TM}}$ System 3 Wireless whiteboard with Bluetooth technology. The receiver is connected via Bluetooth to a computer acting as a server that in turn was connected to a D-Link wireless router enabling three wireless laptops to operate on a local network. Each of the three IW subgroups had a laptop that they used to communicate with the other sub-groups.

Collaborative argumentation tools were designed and integrated into the IW to support collaborative decision-making in medicine. The IW can display in real time not only the representations of the actions of individual role-playing sub-groups, but also those of the observers. Whiteboard diagrams represent what happens in scenarios by displaying content specific information. Patients' information is categorized as brief history, vital signs, prescriptions, and decisions. The structure is similar to the patient's chart in the hospital. Some changes are made in order to make the change of problem space obvious so that students could recognize the pattern of the problem. For example, patient vital signs are put in the middle to highlight the deteriorating situation of the patient. Decisions and prescriptions are marked next to the changing vital signs column to demonstrate the connection of these three kinds of information. Collaborative argumentation tools allow 'observing' students to play an active 
role by annotating, commenting on and suggesting alternatives to decisions of other students. The tools were designed to scaffold collaborative decision-making processes by promoting productive discussions of various proposed actions and plans by providing a structured patient chart where students could comment on the decisions of other participants in the activity and participate by proposing alternative moves (See Fig. 1).

\subsection{Data analysis}

Audio and video records of participant decision-making and communicative activities were collected during two teaching sessions to characterize and compare student behavior in the two situations. Two kinds of data were collected: (a) collaborative decision-making discourse, and (b) computer records of whiteboard annotations. These data were categorized and analyzed from the following four perspectives: (a) decision-making activities, (b) face-to-face communicative activities (c) online annotation (or communication) activities, and (d) the overall relationship among these activities. The analyses will focus on answering the following research questions correspondingly:

1. Do the decision-making activities of TW and IW groups differ?

2. Do the communicative activities of TW and IW groups differ?

3. What are the features of interactive whiteboard annotation?

4. Why do TW and IW groups decision-making and communicative activities differ?

Descriptive analysis was used to identify and characterize differences in TW and IW group decision making (Question 1) and communicative activities (Question 2) and to characterize IW group annotation behavior (Question 3). Qualitative analysis was used to explore the themes and patterns in students' decision making and to see whether they can be explained by the findings from Questions 1 to 3 .

\subsubsection{Do the decision-making activities of TW and IW groups differ?}

In order to investigate whether and how the TW and IW groups differed in their decision-making activities (DMA) in different sessions and in different stages student verbal protocols were collected, transcribed, coded, and compared.

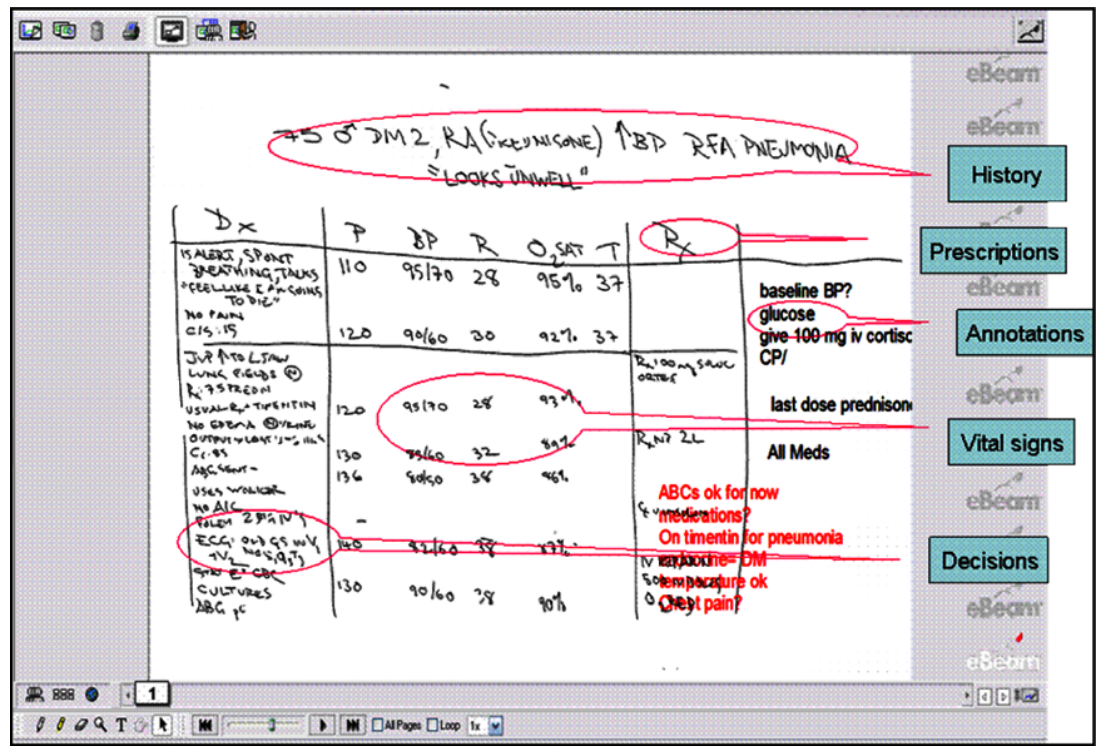

Fig. 1. Screenshot of eBeam whiteboard. 
The literature on ill-structured problem solving and medical decision-making were considered jointly in developing a coding scheme for this task (Chi \& Glaser, 1985; Lajoie et al., 1998; Sinnott, 1989; Voss \& Post, 1988). In this regard, top-level codes were established pertaining to planning and searching for evidence to support a goal in the context of medical decision-making. However, student protocols of the deteriorating patient scenario were analyzed from the bottom-up as well to ensure that the coding covered key features of decision making in emergency medical situations. New codes were established that dealt with the urgency of emergency medicine and included codes pertaining to patient management and stabilizing the patient prior to making other diagnoses. Students were required to make preliminary judgments about a case by interpreting the problem state, as provided in the patient's medical history, and then explain and communicate their decisions to others. The coding categories consisted of: (a) planning, (b) collecting data, (c) managing, and (d) interpreting (see Table 1). The coding schema was reviewed and validated by both the medical teacher and experts in the area of problem solving and discourse analysis. The unit of coding is the meaningful unit of their verbal protocol (Chi, 1997). This unit can be a word, a sentence, or a paragraph.

\subsubsection{Do the communicative activities of TW and IW groups differ?}

Face-to-face communication activities (CA) were coded in order to identify the kinds of activities students used to communicate during the 'deteriorating patient' activity. Student verbal protocols were collected, transcribed and coded to quantify and compare the CA across different sessions and stage between the two groups.

The purpose of the CA coding is to characterize (a) how students express and share their understanding of the deteriorating patient's situation and (b) how they negotiate plans and the actions by which they will solve the patient's medical emergency. CA coding was developed based on the study by van Boxtel (2000) and later incorporated in research on computer-mediated collaboration by Saab and colleagues (Saab, van Joolingen, \& van Hout-Wolters, 2005). Six CA categories were identified: (a) Informative, (b) Argumentative, (c) Elicitative, (d) Responsive, (e) Directive, and (f) Off-task (see Table 2). Subcategories were also identified for some of the categories. For instance, Responsive includes statements of agreement or disagreement between participants. Argumentative includes clarifications of misconceptions, and the organization of understanding through the use of justifications, elaborations and expansions on arguments.

These measures focused on identifying and categorizing the semantic nature of communicative interactions and were designed to determine the effect of group interaction and negotiation on decision-making. The unit of analysis could be any meaningful unit of verbal interaction, such as a word, a sentence, or a paragraph.

Nvivo 2.0, a software program, was used to code verbal data. The Tree of nodes was used to create categories and subcategories that correspond to the meaningful utterances in the coding system identified in Tables 1 and 2. Nvivo provides reports of frequency for each category and subcategories. Only frequencies for first level nodes (the categories) are used for descriptive analysis.

\section{Table 1}

Decision-making activity coding categories

\begin{tabular}{|c|c|c|}
\hline DMA & Definitions & Examples \\
\hline 1. Planning & $\begin{array}{l}\text { Students formulate plans for collecting } \\
\text { patient data and for managing patient } \\
\text { condition }\end{array}$ & $\begin{array}{l}\text { "We should ask the nurse for the vital } \\
\text { signs and at the meantime go to see the } \\
\text { patient ourselves" }\end{array}$ \\
\hline \multirow[t]{2}{*}{ 2. Collecting Data } & $\begin{array}{l}\text { Students collect patient data, i.e., physical } \\
\text { condition, laboratory tests, treatment status }\end{array}$ & "We will be looking for air input first" \\
\hline & & "Does the patient have IV or a foley?" \\
\hline 3. Managing & Students manage patient's condition & "So I would continue with the IV" \\
\hline \multirow[t]{2}{*}{ 4. Interpreting } & $\begin{array}{l}\text { Students interpret patient's condition based } \\
\text { on collected data and management } \\
\text { interventions to construct shared } \\
\text { understandings }\end{array}$ & "He has the history of diabetes" \\
\hline & & $\begin{array}{l}\text { “...because his blood pressure has been } \\
\text { getting lower and lower" }\end{array}$ \\
\hline
\end{tabular}


Table 2

Categories describing communicative activities

\begin{tabular}{|c|c|c|}
\hline Category & Description & Example \\
\hline 1. Informative & Speaker provides information & \\
\hline 1.1 Statement & Communication & "We did not find any obvious signs of bleeding" \\
\hline 2. Argumentative & Utterances may contain the following words & \\
\hline 2.1 Reason & "because" & "We should give oxygen because she's had dyspnea" \\
\hline 2.2 Condition & "if" & "Just to see if there is any, maybe, heart failure" \\
\hline 2.3 Consequence & "then, thus, so" & $\begin{array}{l}\text { "The bolus helped him the first time. So I would bolus } \\
\text { him again" }\end{array}$ \\
\hline 2.4 Continuation & "and, then, so" & "And then we can look at what meds" \\
\hline 2.5 Counter & "but", "no + explanation" & “No, but it won't show on the X-ray" \\
\hline $\begin{array}{l}2.6 \text { Elaborate/ } \\
\text { Expand }\end{array}$ & $\begin{array}{l}\text { A verbalization further explaining a previous } \\
\text { statement }\end{array}$ & "means like, we can see if airway is fine" \\
\hline 2.7 Evaluation & One's opinion or judgment related to the task & "No, that would not be good" \\
\hline 3. Elicitative & Speaker asks for addressee's opinion & \\
\hline 3.1 Question & Asking for information and checking & \\
\hline 3.1.1 & Checking ideas, opinion, or reasoning & "The vital signs?" \\
\hline \multicolumn{3}{|l|}{ Verification } \\
\hline 3.1.2 Open & Asking for new information & “What's our differential?” \\
\hline 3.2 Proposal & Suggestion for a common action & "We go see the patient?" \\
\hline 4. Responsive & Speaker reacts to an earlier utterance & \\
\hline 4.1 Acceptance & Neutral support & “Ok, crash cart” \\
\hline 4.2 Negation & Objection without explanation & “No" \\
\hline 4.3 Confirmation & Explicit support & “Yeah" \\
\hline 4.4 Repeat & Repetition of the previous utterance & "So we're gonna get the vital signs," \\
\hline 5. Directive & $\begin{array}{l}\text { Speaker gives an instruction or makes a } \\
\text { suggestion }\end{array}$ & \\
\hline 5.1 Suggestion & & "We should wait for the X-ray first" \\
\hline 5.2 Order & & "You present the case" \\
\hline 6. Off task & Utterances irrelevant to problem solving task & \\
\hline
\end{tabular}

Two independent raters rated $25 \%$ of all protocols. The percentage of agreement between raters was used for the inter-rater reliability. For DMA, the inter-rater reliability of Planning was $88 \%$, of Collecting data was $90 \%$, of Managing was $79 \%$, and of Interpreting was $87 \%$. For CA, the inter-rater reliability of Informative was $92 \%$, of Argumentative was $86 \%$, of Elicitative was $89 \%$, of Responsive was $84 \%$, and of Directive was $82 \%$.

\subsubsection{What are the features of IW group online annotation?}

Digitized records of whiteboard writings and computer annotations were analyzed to characterize the online argumentation activities. Camtasia Studio, a software program, was used to video record computer screen actions of student laptop actions. Camtasia Studio made it possible to discover how students' verbal data correspond with their whiteboard annotations and laptop entries, thus rendering verbal data more meaningful.

\subsubsection{Why do TW and IW groups decision-making and communicative activities differ?}

A microanalytic method was used to identify and explain differences in decision-making and communicative activities. Microanalytical analysis is used to investigate learning processes in the sociocultural/situative tradition (Teasley \& Roschelle, 1993), whereby social activity, communication, and knowledge representation are inextricably bound together (Schoenfeld, Smith, \& Arcavi, 1993). Such methods have been used to investigate how students construct joint problem spaces and group cognition through the use of cognitive tools (Stahl, 2005).

Classroom discourse was segmented into episodes to examine decision-making and communication patterns for each stage to show the difference between the control and experimental groups and the connections between the decision making and communicative activities between the two groups. Episodes in this discourse were semantic units that are characterized as coherent sequences of sentences (van Dijk, 1981). 


\section{Results}

This section presents the results for each research question.

\subsection{Do decision-making activities of IW and TW groups differ?}

Descriptive analysis is used to compare the decision-making activities of the IW and TW groups in two situations: (a) teaching sessions: refer to the two different sessions for both TW and IW group (1 vs. 2), and (b) decision-making Stage (DMS): Early (subgroup 1[SG1]) or Late (subgroup 2 [SG2] and sub-group 3 [SG3]).

There were no obvious differences in decision-making activities between the two teaching sessions. As to the difference between stages, Fig. 2 revealed differences between the IW and TW groups in terms of Managing and Interpreting. In the early stage of decision-making when the patient's situation was less urgent, the IW group put more effort into Interpreting the situation but less into Managing the patient than the TW group did. In the late stage, when the patient's situation had become more urgent, the IW group put more effort into Managing the patient than into Interpreting the situation.

\subsection{Do communicative activities of TW and IW groups differ?}

The deteriorating patient activity took $30-50 \%$ of each teaching session. During this time, students did not communicate a great deal even when encouraged to do so, perhaps because they were not used to solving problems collaboratively in situations where they were expected to work quickly, carefully and accurately under pressure. As students worked to come up with solutions according to the patient's dynamically changing situation, they tended to try to communicate with the teacher instead of discussing the situation with their peers. Consequently, the number of communication activities was small, compared to the number of decision-making activities. Five CA coding categories were identified. There is no off-task discourse during the role play stage which implied that the students were highly focused on their work while engaged in urgent decision making.

Descriptive methods were used to discover CA patterns between IW and TW conditions. Frequency distributions are listed in Table 3. More utterances were produced in the Early stage by both groups, except that TW group produced more Informative remarks in the Late stage.

\subsection{What are the features of the IW group online annotation?}

Subgroups (SGs) used eBeam to annotate decision-making activities on their laptops while observing another SG stabilize the deteriorating patient. Thus, while SG1 was solving the problem, SG2 and SG3 made annotations and while SG2 was solving the problem, SG1 and SG3 made annotations.

Frequency distribution of DMA across stages

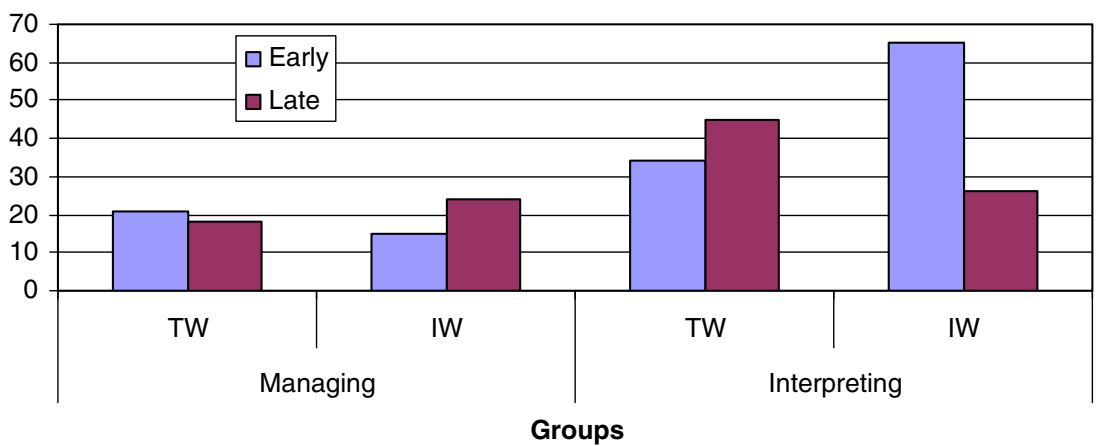

Fig. 2. Frequency distribution of DMA of IW and TW groups across two stages. 
Table 3

Frequency distribution of communicative activities across two stages

\begin{tabular}{llcccrr}
\hline \multirow{2}{*}{ Group } & DMS & \multicolumn{2}{l}{ Communicative activities } & & \\
\cline { 3 - 6 } & & Informative & Argumentative & Elicitative & Responsive & Directive \\
\hline TW & Early & $3(4 \%)$ & $30(41 \%)$ & $13(18 \%)$ & $17(23 \%)$ & $11(14 \%)$ \\
& Later & $6(16 \%)$ & $9(24 \%)$ & $10(26 \%)$ & $10(26 \%)$ & $3(8 \%)$ \\
IW & Early & $19(21 \%)$ & $27(30 \%)$ & $19(21 \%)$ & $18(20 \%)$ & $8(9 \%)$ \\
& Later & $6(11 \%)$ & $8(16 \%)$ & $8(16 \%)$ & $20(39 \%)$ & $9(18 \%)$ \\
\hline
\end{tabular}

Three themes were identified in the annotations: (a) disagreement with other's decision-making actions, (b) proposal for different decision-making actions, and (c) interpretation of the situation. Of all annotations, $83.9 \%$ are Proposals recommending data collection or patient management alternatives, $12.9 \%$ are Interpretations of the situation, and 3.2\% express Disagreements about some action. Annotations were examined with respect to when they occurred and how they influenced decision-making and vice-versa. Annotations were more predominant in the later stage as the patient's situation grew increasingly urgent and decision-makers tended to take suggestions from observer annotations. E.g., in Teaching Session 1 an SG1 student asked about 'medication', 'blood glucose' and 'blood culture' based on annotations provided by SG2 and SG3. This suggests that as the problem becomes more difficult and decision-makers ran out of their own ideas, they become more open to the opinions of observers.

If an observer's annotation was not taken up by decision-makers, they would take it up as soon as their turn came to work on the problem. E.g., the first thing SG2 did when their turn came was to ask about 'lung auscultation' which they had annotated while SG1 was working on the problem. The first thing SG3 did when their turn came was to ask about 'blood glucose' which they had written while SG1 was solving the problem.

\subsection{Why do TW and TW groups decision-making and communicative activities differ?}

Microanalytic methods (as defined above) were used to discover qualitative relationships and mechanisms among decision-making and communication activities. Three episodes were identified in the transcripts that reflect the integration of decision-making and communication activities within and between groups. These episodes are characterized as semantically coherent sequences of sentences differentiating different stages of problem solving. In Episode 1, students discussed background information provided by the teacher about the deteriorating patient and questioned the Nurse about the patient's current situation. This episode pertains to data collection and involves little conflict. TW and IW groups are similar at this episode in terms of the information they discussed, it was either common knowledge, or the information has been introduced by the teacher. Words such as "and", "if", and "then" are commonly used in Argumentative discourse activities. Frequency distributions (Table 3 ) indicated that both TW and IW groups produced more Argumentative discourse in the early stage, $41 \%$ and $30 \%$, respectively. The discourse patterns identified in Episode 1 indicates the production of shared problem-solving knowledge.

Episode 2 began when the teacher initiated the deterioration of the patient forcing the students to stabilize the patient. By causing the patient's vital signs to deteriorate the teacher forced students to take decisive actions that could either help or have no effect. Conflicts started to emerge as to what actions should be taken. Following are two excerpts from TW and IW groups, respectively.

A conflict occurred in the TW group towards the end of SG1's performance after they collected most of the relevant data according to the emergency algorithm. $\mathrm{T}$ is the teacher, and $\mathrm{O}$ and $\mathrm{M}$ are students.

T: Bolus of NS? Yes, doctor? How much would you like to give him? (The teacher acted as the nurse and inquired about how to bolus the patient.)

0 : Uh, five hundred.

M: Would you be...

O: (interrupts $M$ ) Listen to the heart before, listen to heart before? 
M: Just to see if there is any, maybe, heart failure. In that case we would have to be careful if we give any saline. (because) We do not want to overload the [patient].

Towards the end of the early stage of problem solving, the TW group started to exchange opinions about collecting data and patient management. Even though there were conflicts, students tended to reach agreements with little negotiation. The discourse revealed more Elicitative and Responsive utterances indicating simple acceptance rather than a negotiation of opinions.

When the IW group was at a similar stage in problem solving, SG1 M and P ran into the same difficulty toward the end of their turn and they consulted the annotations and began a discussion.

M: Another bolus? OK, Glucose. Another bolus. And do we want to... Ah meds? He could be taking sort of (meds). That could (cause the problem). Has he taken an antibiotic lately that could have... Sounds medications. Complete blood count. OK, is that alright?

P: Hmmm.

M: Sounds like medications could play a thing?

P: Hmmm.

M: Is that alright?

P: Before that we should bolus him again?

M: We should bolus him again. The bolus helped him the first time. So I would bolus him again.

$P$ : And then we can look at what meds he is taking.

$P$ and $M$ contributed equally to the dialogue about what data to collect and how to manage the patient. Shared understanding of the situation is achieved by communication. This phenomenon is consistent with the findings demonstrated in Table 3 which indicated that IW group discourse was more informative in the early stage of problem solving.

In Episode 3, with the increasing deterioration of the patient, both IW and TW group had difficulty communicating because they did not know how to proceed. IW group's discourse topics, were often triggered by outside opinions in the form of annotations. Although students came into conflict they also tried to justify their opinions in order to reach consensus.

TW and IW groups demonstrated similarities and differences at various episodes in the activity. Both groups engaged in short, superficial discussions to build shared understandings of the patient's situation during the early stage. During the middle stage, both groups argued about how best to solve the patient's problem. TW group quickly reached agreement on data collection and patient management but with one student dominating the discourse whereas IW group reached agreement through equal contributions which implied that they had constructed shared understandings through communication. In the late stage, both groups ran into difficulty solving the case. TW group engaged in few decision-making activities and their discourse exhibited little argumentation or elaboration. IW group produced relatively more decision-making acts which were often triggered by interactive whiteboard annotations. The sections below will further characterize the content and pattern of communication to explain the difference in TW and IW groups.

\subsubsection{Content of communication}

Student behavior while using the interactive whiteboard can be characterized in terms of their verbal discourse. During the early stage of decision-making when there was no imminent pressure of the deterioration of the patient, SG1 of IW group asked many questions relating to the patient's situation and tried to identify possible reasons for the patient's deteriorating condition. However, because they were not on the right track in their questioning, the patient's vital signs kept on deteriorating. When the teacher asked 'What are you going to do?' the students responded 'Not really going to say' which indicated that they did not have further ideas. The students then referred to the annotations on their laptops, but they were not sure what they were supposed to do, so they asked the teacher 'We can see all their comments?' The teacher said 'I know, I know that's the whole point' to confirm their inquiry. The above discourse demonstrated that initially students were not clear about the purpose of the tools but after this exchange students' decision-making and discourse could have been influenced by the annotations.

Later on, the students' decision-making activities as indicated by verbal interactions were directly or indirectly influenced by the interactive whiteboard annotations. SG1 students began asking ques- 
tions based on such SG3 annotation as "what meds he's on?'. One medication he was on was "prednisone" and this information influenced their later interaction. SG1 students discussed whether the prednisone should be discontinued. P (in SG1) proposed stopping Prednisone, while M (in SG1) was worried about whether stopping steroids too suddenly would result in the patient going 'cold turkey', an acute withdrawal reaction that can lead to a crisis situation. A large stretch of the following conversation focused on this topic and was mainly informative and argumentative. These examples support the frequency distribution results in Table 3 which showed that in the early stage, IW group has more informative and argumentative utterances. From a decision-making perspective, it is obvious here that most of the activity involved interpreting the situation. This explains the results in Table 3 that in the early stage (SG1) of decision-making, IW group showed significantly more Interpreting activity which is supported by informative and argumentative behavior.

SG2 was mainly concerned about checking the lungs. SG2 wrote on the interactive whiteboard 'listen to the lungs' before their turn came, and SG3 wrote ' $C X R / C$ ' which means chest X-ray. The whole discussion in this teaching session was about checking the lungs. Their first question 'have you listened to his lungs' reflected their concern about this issue since SG1 failed to deal with the lungs. The only other question asked was 'fluid intake' which was also annotated by SG2. A similar pattern was also seen with SG3 whose data collecting was directed by their earlier annotations or by those of other subgroups. Management actions were also influenced by the opinions of others, which gave rise to discussions and reflections (interpretation).

It is obvious that SGs took the interactive whiteboard annotations into consideration in the later stages of decision-making, especially in Teaching Session 2. Suggestions for collecting data and managing the patient were taken into consideration and this may explain why the IW group spent more time managing the patient than the TW group in the later stage of the teaching session.

Pattern of interactions. This section describes pattern of interactions that characterize differences between the TW and IW group. During the deteriorating patient activity, students were always encouraged to discuss problems themselves. However, when they encountered a problem they rarely talked about it. This was more obvious in the TW group. For example in Teaching Session 1, the teacher gave the TW group explicit instructions to discuss problems among themselves. Students B and G had different plans for the patient, B told the nurse to check the patient's 'ECG' and G proposed giving the patient 'Dextrose'. Here is an example.

T: You better discuss among yourselves. I mean one of you is telling me dextrose and the other is telling me ECG. You guys have to decide.

(B \& G Confer)

B: So? (To G)

G: Is dextrose ok with you guys? (To B) 5 percent with saline.

$B$ : Yes, I think we could give him dextrose.

$B$ and $G$ had a brief communication after the teacher's instructions because they had different proposals for the patient. B asked G's opinion who then gave his proposal which B immediately accepted. A similar pattern occurred later on when $G$ and B talked about giving more liquid.

T: More bolus? Discuss among yourselves.

$G$ : Can we give more bolus? Just feel like he maybe crash.

$B$ : Yes, could give bolus and [...] after

$G$ : And actually, at the meantime we're cross matching some blood.

T: So what you are saying is get off a cross match, ok, and we will give another bolus of what?

$B$ : Dextrose.

G: Can we bolus with dextrose? (talk to B)

$B:$ No.

G: No, that would not be good.

The discussion was brief with little argumentation. Even though G and B disagreed initially, they came to an agreement with little negotiation. The same pattern could be seen in the rest of the problem solving episodes. 
As demonstrated above, TW group interactions were brief and involved little negotiation and elaboration. Their discussion was not spontaneous and usually needed teacher's instruction. IW group discussions involved more turn taking and students contributed more equally in terms of offering ideas. IW group students seemed to express their opinions and the teacher seldom interrupted or pushed them to collaborate. Their discussions were often based on ideas provided by annotations (see example of IW group in Episode 2).

$\mathrm{M}$ read the annotations and repeated, i.e., 'glucose', 'another bolus', and 'meds.' $\mathrm{M}$ then began elaborating possible reasons for the problem, such as taking inappropriate medication. P agreed and suggested that they 'access his chart'. M then proposed to bolus the patient again. P did not object but insisted that they should also check the patient's medications.

This study investigated the collaborative decision-making and communicative discourse of two groups of learners that were engaged in a simulated medical emergency in two conditions. Descriptive analysis showed the IW group's decision-making behavior is more adaptive in the context of medical emergencies because early shared understanding led to subsequent effective patient management. Given the need to act quickly in medical emergencies facilitating early communication is important to saving patient lives. Qualitative analysis revealed that the IW and TW groups showed different decision-making and communication integration across time. The IW promoted different patterns of collaborative discourse and productive argumentation. It is concluded that this particular use of technology facilitated the construction of shared situation models and joint problem spaces that led to more adaptive performance in an emergency situation.

\section{Discussion}

The delivery of emergency medical care typically involves teams of medical professionals working in highly organized technological and institutional environments. Research on collaborative NDM shows that decision-makers rely on (a) shared task models, (b) effective communication skills, (c) acute situational awareness, and (d) meta-cognitive skills (Orasanu, 2005). Shared task models are composed of highly integrated jointly held systems of knowledge (Orasanu \& Salas, 1993). For instance, as medical emergency situations develop so must the complexity of the shared understandings that enable medical teams to collaborate effectively. Collaborators need effective communication skills to co-construct the shared understandings of common goals, plans and actions for making patient management decisions. Collaborators must communicate quickly and accurately among themselves and with other medical professionals about patients' rapidly changing medical condition. Meta-cognitive skills are essential in situations where collaborators have to know what needs to be done and what resources and forms of expertise are needed (Orasanu, 2005) because they enable students to reflect on their problem solving abilities to seek assistance when needed.

In this study a comparison of IW and TW group discourse reveals that shared annotations guide IW group communicative activities leading to more productive decision-making activities. The findings suggest that interactive whiteboard enabled IW group to share data and to construct shared understandings about the patient. Such shared visualization can clarify verbal interaction, promote productive argumentation and facilitate negotiation. In the early stage of the simulations the IW group spent more time interpreting the patient's history, laboratory tests, and vital signs and in the later stage of the simulation, as the patient's situation grew increasingly dire, the IW group engaged in more management actions to stabilize the patient. In contrast, the TW group interpreted less in the early stage of the simulation but more in the later stage but engaged in few patient management actions in the later stage. In emergency medical care physicians seek to stabilize patients rather than to diagnose them. For instance, when a patient stops breathing the physician needs to ventilate them before finding out why. Thus, given that IW group did more to manage the patient than the TW group, IW group's performance is more adaptive to emergency medicine. Shared cognition facilitates the construction of shard situation models and joint problem spaces which lead to better decision making and problem solving.

Based on the findings from communicative discourse, both TW and IW groups communicated more in the early stage of the simulation. The decrease of communication over time has been the result of 
the increased urgency of the patient's situation and the difficulty of the task. Kleinman and Serfaty (1989) found that communication decreased as workload increased. They interpreted this decrease as a function of mutual mental models emerging over time that allowed participants to anticipate each other's resource needs and actions.

These results support the hypothesis that the argumentation tools embedded into the IW design enhance group decision-making and communicative interactions in the simulated medical emergency, the deteriorating patient activity. In the early stage of the activity the IW group communicated to construct a better understanding of the deteriorating patient. Part of their communication was supported by the shared access to the patient chart that was dynamically annotated by each group as they made decisions. These annotations were shared via the IW technology that allowed each group to annotate the patient chart on their own laptop and share the information with others when done. Anything that was inscribed on the TW by the teacher was recorded dynamically as the vital signs of the deteriorating patient changed with student interactions. Each individual IW subgroup accessed and interacted with this patient chart information via their laptops. The ability to view and interact with this information supported the groups' communicative interactions allowing them to propose plans, suggest alternatives, seek assistance, and reflect and comment on each other's ideas. The visualization of shared information facilitated communicative interactions and decision-making of the IW group in the early stage of the simulation. Although both the IW and TW groups have visual access to the patient chart on the TW, the IW subgroups had personalized visual access of the same patient chart and were able to inscribe and share annotations via their IW equipped laptops. IW subgroups observing the role-playing subgroup can still make decisions by annotating the patient chart on their personal IWs. Thus, whether the IW subgroups are observing or make decisions they still share information and ultimately decisions. Sharing whiteboard annotations stimulated productive problem solving by documenting previous decisions allowing teams to reflect on the information that influenced their subsequent decisions.

This study investigated the role of shared understanding by investigating the relationship between technology, argumentation, and cognitive processes. It is proposed that an argumentative approach is necessary for solving ill-structured problems, especially when argumentation can be visualized and shared (van Bruggen, Boshuizen, \& Kirschner, 2003). Even though the interpretations and strategies used for solving problems in such time emergent situations are very concise, the findings of this study show that the ability to visualize and share argumentation help to construct, interpret and focus on shared representations of problems which are essential for collaborative problem solving (Alpay, Giboin, \& Dieng, 1998).

The situations described in this article demonstrate collaborative NDM activities that build on shared mental and situation models that are consistent with the theory of 'common ground'(Clark \& Brennan, 1991). Collaborators in face-to-face decision-making, use both verbal and non-verbal discourse to construct shared mental and situational models or understandings of the environment, the task and team knowledge (Luczak, Muhlfelder, \& Schmidt, 2003). In this study, environmental understandings encompassed understanding the technology and cognitive tools (Lajoie, 2000; Orasanu, 2005; Salas, Oser, Cannon-Bowers, \& Daskarolis-Kring, 2002) designed to support the teaching activity. Team knowledge includes shared understanding about team interaction processes, such as sharing information, monitoring each other's activities and solutions, and supporting each other. Members of an effective team provide information without being asked because they know it is needed because they have reflected on shared task understandings (Serfaty, Entin, \& Volpe, 1993). This phenomenon has been called 'cognitive empathy' (Artman \& Waern, 1998). Results showed that IW group engaged in more informative interactions in the early stage while TW group engaged in more informative interactions in the later stage. Meanwhile, IW group engaged in other discourse activities in the early stage as well. Compared to TW group, IW group built a shared understanding about the patient situation and the task in the early stage, which enables the team to work more efficiently and effectively. This is consistent with the hypothesis that shared visualization will facilitate communicative interactions and the development of shared understandings.

This study took the clinical teaching session as the research environment and as such it was limited by constraints inherent to this teaching environment. The study could not be controlled as well as a lab-based setting. Although we can assume comparable background knowledge of the participants given the strict entry requirements to medical school and that bias was somewhat reduced by randomly 
assigning the groups to the TW and IW conditions, there are still student attributes that were not controllable. For example, attributes such as personality, openness to ideas, communication skills, etc., may have played a role in determining the results in the two conditions. The results indicate that sharing annotations in the structured patient chart facilitate the shared understanding of the patient's situation and management. However, we still need to determine which mechanisms afforded by the structured IW activity led to positive results. In the future, better-controlled studies can be designed to differentiate which annotation functions lead to significant findings: structuredness, content of annotation, or sharedness.

Theory-based technology tools can lead to learning environments where students interact with data in dynamic ways that are different than instructional delivery systems (Kozma, 1991; Lajoie, 2007). In this respect, argumentation tools were designed to support naturalistic decision-making within a pedagogical situation that required collaboration in a problem-solving context. Argumentation tools were designed to be content specific and consequently helped constrain the decision making process by scaffolding the process. Future work can examine the effectiveness of these tools in an extended clinical teaching semester to see how the quality of arguments and decisions change over an extended period of time. Another direction will be to examine the use of such tools in distributed environments sharing the deteriorating patient simulation with students at a distance. With interactive whiteboard support and networked computers, students from different teaching hospitals can benefit from such learning activities. Similarly, interactive whiteboards can be used in clinical practice where distributed expertise is required. In medical decision-making, complex clinical cases often call for the coordination and collaboration of medical professionals with different forms of expertise in order to achieve a shared goal, such as patient recovery. Interactive whiteboards enable teams of medical professionals to visualize, annotate and share patient information even when some members of the team are not physically present. By enabling teams of physically and virtually present experts to collaboratively access information, visualize and share ideas, and establish effective communication, cognitive tools such as interactive whiteboards can promote medical problem solving. This case study shows clear ways in which cognitive tools, such as interactive whiteboards that are structured to assist in documenting a medical argument, can enable teams of medical professionals to collaboratively leverage their problem solving expertise.

\section{References}

Alpay, L., Giboin, A., \& Dieng, R. (1998). Acidentology: An example of problem solving by multiple agents with multiple representations. In M. W. v. Someren, P. Reimann, H. P. A. Boshuizen, \& T. d. Jong (Eds.), Learning with multiple representations (pp. 152-174). Amsterdam: Pergamon.

Andriessen, J., Baker, M. J., \& Suthers, D. (2003). Argumentation, computer support, and the educational context of confronting cognition. In J. Andriessen, M. J. Baker, \& D. Suthers (Eds.), Arguing to learn: Confronting cognitions in computer-supported collaborative learning environment (pp. 1-25). Dordrecht, Netherlands: Kluwer Academic Publishers.

Artman, H., \& Waern, Y. (1998). Creation and loss of cognitive empathy at an emergency control centre. In Y. Waern (Ed.), Cooperative process management: Cognition and information technology (pp. 69-76). London: Taylor \& Francis.

Baker, M. (1994). A model for negotiation in teaching-learning dialogues. Journal of Artificial Intelligence in Education, 5 , 199-254.

Baker, M. (2003). Computer-mediated argumentative interactions for the co-elaboration of scientific notions. In J. Andriessen, M. Baker, \& D. Suthers (Eds.), Arguing to learn: Confronting cognitions in computer-supported collaborative learning (pp. 47-78). Dordrecht, Netherlands: Kluwer.

Baker, M., \& Lund, K. (1997). Promoting reflective interactions in a CSCL environment. Journal of Computer Assisted Learning, 13, 175-193.

Baker, M. J. (1995). Negotiation in collaborative problem-solving dialogues. In R. J. Beun, M. J. Baker, \& M. Reiner (Eds.), Dialogue and instruction: Modeling interaction in intelligence tutoring system (pp. 39-55). Berlin: Springer.

Beach, L. R., \& Lipshitz, R. (1993). Why classical decision theory is an inappropriate standard for evaluating and aiding most human decision making. In G. A. Klein, J. Orasanu, R. Calderwood, \& C. E. Zsambok (Eds.), Decision making in action: Models and methods (pp. 21-35). Westport, CT: Ablex Publishing.

Brown, J. S., Collins, A., \& Duguid, P. (1989). Situated cognition and the culture of learning. Educational Researcher, 18(1), 32-42.

Cannon-Bowers, J. A., \& Salas, E. (Eds.). (1998). Making decisions under stress: Implications for individual and team training. Washington, DC: American Psychological Association.

Cannon-Bowers, J. A., Salas, E., \& Converse, S. (1993). Shared mental models in expert team decision making. In N. J. J. Castellan (Ed.), Individual and group decision making (pp. 221-246). Hillsdale, NJ: Lawrence Erlbaum.

Carr, C. S. (2003). Using computer supported argument visualization to teach legal argumentation. In P. A. Kirschner, S. J. Buckingham Shum, \& C. S. Carr (Eds.), Visualizing argumentation: Software tools for collaborative and educational sense-making (pp. 75-96). London, UK: Springer. 
Chan, C. K. K., Burtis, J., \& Beretier, C. (1997). Knowledge building as a mediator of conflict in conceptual change. Cognition and Instruction, 15, 1-40.

Chi, M. T. H. (1997). Quantifying qualitative analyses of verbal data: A practical guide. Journal of the Learning Sciences, 6, 271-315.

Chi, M. T. H., \& Glaser, R. (1985). Problem solving ability. In R. J. Sternberg (Ed.), Human abilities: An information processing approach (pp. 227-250). New York: W.H. Freeman and Company.

Chi, M. T. H., Slotta, J. D., \& de Leeuw, N. (1994). From things to processes: A theory of conceptual change for learning science concepts. Learning and Instruction, 4, 45-69.

Chi, M. T. H., \& VanLehn, K. A. (1991). The content of physics self-explanations. Journal of the Learning Sciences, 1, 69-105.

Chong, S. M. (1998). Models of asynchronous computer conference for collaborative learning in large college class. In C. J. Bonk \& K. S. King (Eds.), Electronic collaborators (pp. 157-182). Mahwah, NJ: Lawrence Erlbaum.

Clark, H. H., \& Brennan, S. E. (1991). Grounding in communication. In Lauren B. Resnick, John M. Levine, \& S. D. Teasley (Eds.), Perspectives on socially shared cognition (pp. 127-149). Washington, DC: American Psychological Association.

Dosie, W., \& Mugny, W. (1984). The Social development of the intellect. Oxford: Pergamon Press.

Edelson, D., \& O'Neill, D. K. (1994). The CoVis collaboratory notebook: Supporting collaborative scientific enquiry. Paper presented at the Annual National Educational Computing Conference, Boston, Massachusetts.

Fischer, F., Bruhn, J., Grasel, C., \& Mandl, H. (2002). Fostering collaborative knowledge construction with visualization tools. Learning and Instruction, 12, 213-232.

Gaba, D. (1992). Dynamic decision-making in anesthesiology: Cognitive models and training approaches. In D. A. Evans \& V. L. Patel (Eds.), Advanced models of cognition for medical training and practice (pp. 123-148). Heidelberg, Germany: Heidelberg.

Guzdial, M., \& Turns, J. (2000). Effective discussion through a computer-mediated anchored forum. Journal of the Learning Sciences, 9, 437-469.

Hirsch, L., Saeedi, M., Cornillon, J., \& Litosseliti, L. (2004). A structured dialogue tool for argumentative learning. Journal of Computer Assisted Learning, 20, 72-80.

Hoadley, C. M., \& Linn, M. C. (2000). Teaching science through online, peer discussions: SpeakEasy in the knowledge integration environment. International Journal of Science Education, 22, 839-857.

Hron, A., Hesse, F. W., Cress, U., \& Giovis, C. (2000). Implicit and explicit dialogue structuring in virtual learning groups. British Journal of Educational Psychology, 70, 53-64.

Jermann, P., \& Dillenbourg, P. (2003). Elaborating new arguments through a CSCL script. In J. Andriessen, M. Baker, \& D. Suthers (Eds.), Arguing to learn: Confronting cognitions in computer-supported collaborative learning environments (pp. 205-226). Dordrecht, Netherlands: Kluwer.

Jermann, P., Soller, A., \& Muehlenbrock, M. (2001). From mirroring to guiding: A review of state of the art technology for supporting collaborative learning. Paper presented at the First European conference on Computer-supported collaborative learning, Maastricht, Netherlands.

Johnson, D. W., \& Johnson, R. T. (Eds.). (1999). Learning together and alone: Cooperative, competitive, and individualistic learning (5th ed.). Boston, MA: Allyn \& Bacon.

Jonassen, D. H. (1995). Computers as cognitive tools: Learning with technology, not from nechnology. Journal of Computing in Higher Education, 6(2), 40-73.

Jonassen, D. H. (2000). Computers as mindtools for school: Engaging critical thinking. Upper Saddle River, N.J: Merrill.

Jonassen, D. H., \& Carr, C. (2000). Mindtools: Affording multiple knowledge representations for learning. In S. P. Lajoie (Ed.), Computers as cognitive tools II: No more walls: Theory change, paradigm shifts and their influence on the use of computers for instructional purposes (pp. 165-196). Mahwah, NJ: Lawrence Erlbaum.

Jonassen, D. H., \& Reeves, T. C. (1996). Learning with technology: Using computers as cognitive tools. In D. H. Jonassen (Ed.), Handbook of research for educational communications and technology (pp. 693-719). New York: Simon and Schuster.

Kanselaar, G., Erkens, G., Andriessen, J., Prangsma, M., Veerman, A., \& Jaspers, J. (2003). Designing argumentation tools for collaborative learning. In P. A. Kirschner, S. J. Buckingham Shum, \& C. S. Carr (Eds.), Visualizing argumentation: Software tools for collaborative and educational sense-making (pp. 51-73). London, UK: Springer-Verlag.

Klein, G., Calderwood, R., \& Clinton-Cirocco, A. (1986). Rapid decision making on the fire ground. Paper presented at the 30th annual meeting of the Human Factors and Ergonomics Society, Santa Monica, CA.

Kleinman, D. L., \& Serfaty, D. (1989). Team performance assessment in distributed decision making. Paper presented at the first conference of Interactive Networked Simulation for Training Orlando, FL.

Kozma, R. (1991). Learning with media. Review of Educational Research, 61(2), 179-212.

Kuhn, D. (1991). The skills of argument. Cambridge: University Press.

Lajoie, S. P. (1993). Computer environments as cognitive tools for enhancing learning. In S. P. Lajoie \& S. J. Derry (Eds.), Computers as cognitive tools (pp. 261-288). Hillsdale, NJ: Erlbaum.

Lajoie, S. P. (2007). Aligning theories with technology innovations in education. British Journal of Educational Psychology Monograph Series II: Learning through digital technologies, 5, 27-38.

Lajoie, S. P. (Ed.). (2000). Computer as cognitive tools: No more walls. Mahwah, NJ: Lawrence Erlbaum Associates.

Lajoie, S. P., \& Azevedo, R. (2000). Cognitive tools for medical informatics. In S. P. Lajoie (Ed.), Computers as cognitive tools: No more walls (pp. 247-271). Mahwah, NJ: Lawrence Erlbaum.

Lajoie, S. P., \& Azevedo, R. (2006). Teaching and learning in technology-rich environments. In P. Winne \& P. Alexander (Eds.), Handbook of educational psychology (2nd ed., pp. 803-821). Mahwah, NJ: Erlbaum.

Lajoie, S. P., Azevedo, R., \& Fleiszer, D. M. (1998). Cognitive tools for assessment and learning in a high Information flow environment. Journal of Educational Computing Research, 18(3), 205-235.

Lajoie, S. P., Garcia, B. C., Berdugo, G. C., Marquez, L., Espíndola, S., \& Nakamura, C. (2006). The creation of virtual and face-to-face learning communities: An international collaborative experience. Journal of Educational Computing Research, 35, 163-180.

Lave, J. (1991). Situated learning in communities of practice. In L. B. Resnick, J. M. Levine, \& S. D. Teasley (Eds.), Perspectives on socially shared cognition (pp. 63-82). Washington, DC: American Psychological Association.

Leprohon, J., \& Patel, V. L. (1995). Decision-making strategies for telephone triage in emergency medical services. Medical Decision Making, 15, 240-253. 
Luczak, H., Muhlfelder, M., \& Schmidt, L. (2003). Group task analysis and design of computer-supported cooperative work. In E. Hollnagel (Ed.), Handbook of cognitive task design (pp. 99-127). Mahwah, NJ: Lawrence Erlbaum.

Nussbaum, E. M., \& Sinatra, G. M. (2003). Argument and conceptual engagement. Contemporary Educational Psychology, 28, 384-395.

Orasanu, J. (2005). Crew collaboration in space: A naturalistic decision-making perspective. Aviation, Space, and Environmental Medicine, 76(6 (Sect. 2, Suppl.)), B154-B163.

Orasanu, J., \& Salas, E. (1993). Team decision making in complex environments. In G. A. Klein, J. Orasanu, R. Calderwood, \& C. E. Zsambok (Eds.), Decision making in action: Models and methods (pp. 327-345). Norwood, NJ: Ablex Publishing..

Piaget, J. (1970). Structuralism (C. Maschler, Trans.). New York: Basic Books.

Reimann, P. (2003). How to support groups in learning: More than problem solving. Paper presented at the 11th International Conference on Artificial Intelligence in Education Sydney, Australia.

Resnick, L., Levine, J., \& Teasley, S. (Eds.). (1991). Perspectives on socially shared cognition. Washington DC: APA Press.

Roschelle, J., \& Teasley, S. D. (1995). The construction of shared knowledge in collaborative problem solving. In C. E. O'Malley (Ed.), Computer-supported collaborative learning (pp. 69-97). New York: Springer.

Saab, N., van Joolingen, W. R., \& van Hout-Wolters, B. H. (2005). Communication in collaborative discovery learning. British Journal of Educational Psychology, 75, 603-621.

Salas, E., Oser, R. L., Cannon-Bowers, J. A., \& Daskarolis-Kring, E. (2002). Team training in virtual environments: An event-based approach. In K. M. Stanney (Ed.), Handbook of virtual environments: Design, implementation, and applications. Human factors and ergonomics (pp. 873-892). Mahwah, NJ: Lawrence Erlbaum.

Salomon, G. (Ed.). (1993). Distributed cognition: Psychological and educational considerations. New York, NY: Cambridge University Press.

Salomon, G., Perkins, D. N., \& Globerson, T. (1991). Partners in cognition: Extending human intelligence with intelligent technologies. Educational Researcher, 20(3), 2-9.

Scardamalia, M., \& Bereiter, C. (1996). Computer support for knowledge-building communities. In T. Koschmann (Ed.), CSCL: Theory and practice of an emerging paradigm: Vol. 1. Computers, cognition, and work (pp. 249-268). Mahwah, NJ: Lawrence Erlbaum.

Schoenfeld, A. H., Smith, J. P., \& Arcavi, A. (1993). Learning: The microgenetic analysis of one student's evolving understanding of a complex subject matter domain. In R. Glaser (Ed.). Advances in instructional psychology (Vol. 4, pp. 55-175). Hillsdale, NJ: Lawrence Erlbaum.

Schwarz, B. B., \& Glassner, A. (2003). The blind and the paralytic: supporting argumentation in everyday and scientific issues. In Jerry Andriessen, Michael Baker, \& D. D. Suthers (Eds.), Arguing to learn: Confronting cognition in computer-supported collaborative learning environments (pp. 227-260). Dordrecht: Kluwer Academic Publishers.

Serfaty, D., Entin, E., \& Volpe,C. E. (1993). Adaptation to stress in team decision making and coordination. Paper presented at the Human Factors and Ergonomics Society 37th Annual Meeting, Seattle, WA.

Sinnott, J. D. (1989). A model for solution of ill-structured problems: Implications for everyday and abstract problem solving. In J. D. Sinnott (Ed.), Everyday problem solving: Theory and applications (pp. 72-99). New York, NY: Praeger.

Slavin, R. E. (1995). Cooperative learning: Theory, research and practice (2nd ed.). Needham Heights, MA: Allyn \& Bacon.

Stahl, G. (2005). Group cognition in chat: Methods of interaction/Methodologies of analysis. Paper presented at the Nordic Analysis of Interaction and Learning, Gothenburg, Sweden.

Stoyanova, N., \& Kommers, P. (2002). Concept mapping as a medium of shared cognition in computer-supported collaborative problem solving. Journal of Interactive Learning Research, 13, 111-133.

Suthers, D., \& Hundhausen, C. (2001). Learning by constructing collaborative representations: An empirical comparison of three alternatives. Paper presented at the First European Conference on Computer-Supported Collaborative Learning, Maastricht, Netherlands.

Teasley, S. D., \& Roschelle, J. (1993). Constructing a joint problem space: The computer as a tool for sharing knowledge. In S. P. Lajoie \& S. J. Derry (Eds.), Computers as Cognitive Tools (pp. 229-258). Hillsdale, NJ: Lawrence Erlbaum.

van Boxtel, C. (2000). Collaborative concept learning: Collaborative learning tasks, student interaction, and the learning of physics concepts. Unpublished Doctoral thesis, Utrecht University, Utrecht, Netherlands.

van Boxtel, C., \& Veerman, A. (2001). Diagram-mediated collaborative learning: Diagrams as tools to provoke and support elaboration and argumentation. Paper presented at the first European Conference on Computer-Supported Collaborative Learning, Maastricht, Netherlands.

van Bruggen, J. M., Boshuizen, H. P. A., \& Kirschner, P. A. (2003). A cognitive framework for cooperative problem solving with argument visualization. In Paul A. Kirschner, Simon J. Buckingham, \& C. S. Carr (Eds.), Visualizing argumentation: Software tools for collaborative and educational sense-Making (pp. 261-267). London: Springer-Verlag.

van Dijk, T. A. (1981). Episodes as units of discourse analysis. In D. Tannen (Ed.), Analyzing discourse: Text and talk (pp. 177-195). Georgetown: Georgetown University Press.

Veerman, A. (2003). Constructive discussions through electronic dialogue. In J. Andriessen, M. Baker, \& D. Suthers (Eds.). Arguing to learn: Confronting cognitions in computer-supported collaborative learning environments (Vol. 1, pp. 117-143). Dordrecht, the Netherlands: Kluwer Academic Publishers.

Voss, J. F., \& Post, T. A. (1988). On the solving of ill-structured problems. In M. T. H. Chi, R. Glaser, \& M. J. Farr (Eds.), The nature of expertise (pp. 261-285). Hillsdale, NJ: Lawrence Erlbaum.

Wang, Y., \& Chen, X. (2006). Improving online learning experience with synchronous chat. Paper presented at the International Conference on Educational Technology, Calgary, Canada.

Webb, N. M., Troper, J. D., \& Fall, R. (1995). Constructive activity and learning in collaborative small groups. Journal of Educational Psychology, 87, 406-423.

Winne, P. H. (2006). How software technologies can improve research on learning and bolster school reform. Educational Psychologist, 41, 5-17.

Zsambok, C. E. (1997). Naturalistic decision making: Where are we now? In C. E. Zsambok \& G. Klein (Eds.), Naturalistic decision making (pp. 3-16). Mahwah, NJ: Lawrence Erlbaum. 